\title{
Possible Chaotic Motions in a Feedback Controlled 2R Robot
}

\author{
Shrinivas Lankalapalli* Ashitava Ghosal ${ }^{\dagger}$ \\ Robotics \& CAD Laboratory \\ Department of Mechanical Engineering \\ Indian Institute of Science \\ Bangalore 560 012, INDIA
}

\begin{abstract}
The equations describing the motion of a feedback controlled robot are known to be non-linear. Several non-linear equations are known to exhibit chaos for certain ranges of parameters. In this paper, we explore the possibility of chaos in a system of differential equations which model a feedback controlled two link robot with rotary $(R)$ joints. We examine a simple proportional and derivative controller and a model based controller for a $2 R$ planar robot undergoing repetitive motions in a plane in the absence of gravity. We show that the differential equations describing such a system exhibits chaotic behavior for certain ranges of the proportional and derivative gains and for certain values of a parameter which quantifies the mismatch between the model and the actual system. We discuss the difficulty of obtaining analytical results and describe numerical schemes to test for chaos and to obtain ranges of gains and the mismatch which results in chaotic motions.
\end{abstract}

\section{Introduction}

Chaotic motions are a class of motions in deterministic physical and mathematical systems whose time history has sensitive dependence on initial conditions[1]. The sensitive dependence implies a divergence of slightly perturbed trajectories and hence long term unpredictability. These type of motions occur in nonlinear differential equations for certain parameters, certain initial conditions and for repetitive motions.

The techniques to obtain the dynamic equations of motion for a serial robot are very well known[2]. The dynamics of a serial robot is described by a set of second-order, non-linear differential equations. The number of such equations is same as the number of degrees of freedom (usually a robot is assumed to have at least two degrees of freedom). There are several

*Graduate Student email: srini@mecheng.iisc.ernet.in

$\dagger$ Assoc. Professor email: asitava@mecheng.iisc.ernet.in non-linear terms in the equations of motion. To name a few, a) the inertia of the robot links are dependent on its configuration giving rise to trigonometric terms in joint rotations, b) the coupling between the motion of the links gives rise to non-linear terms involving trigonometric terms in the joint rotations and quadratic terms in the joint velocities, c) the gravity terms contain trigonometric quantities in joint variables. In addition, for more realistic modeling, nonlinearities due to the flexibility of links and joints, friction at the joints, and non-linearities at the actuators and controller are often taken into account. These nonlinearities form the necessary condition for chaotic behavior. In this paper, we look at the simplest possible rigid body equations of motion containing only the inherent trigonometric non-linearities and non-linearities due to the coupling of motion of the links of a simple planar $2 \mathrm{R}$ robot. We also assume that robot motion is in a horizontal plane and the gravity terms are zero.

Although there exists a vast body of literature on chaotic motions in Duffing's oscillator, inverted pendulum, maps and several other systems[3], there are very few works on chaos in robots reported in literature. Striet et al.[4] have looked at the non-linear response of a flexible manipulator performing repetitive tasks. They show that the flexible variables can undergo period doubling bifurcations leading to chaos. Bühler and Koditschek [5] have discussed robotic juggling and have shown that incrementing controller gains of a planar juggling robot can lead to period doubling and chaotic motions. Vakakis and Burdick[6]and M'Closkey and Burdick[7] have looked at periodic and chaotic motions in a hopping robot with a non-linear spring in the leg. Mahout et al. $[8,9]$ have numerically studied the equations of a planar $2 \mathrm{R}$ robot with a proportional and derivative(PD) controller. They have shown that the $2 \mathrm{R}$ robot, under $\mathrm{PD}$ control, can exhibit harmonic, subharmonic, higher harmonic, fractional harmonic and possible chaotic motions. Except $[8,9]$, to the best of our knowledge, there is no discussion on possible chaotic motions in feedback controlled robot. 
In this paper we study a rigid, planar, $2 \mathrm{R}$ manipulator under feedback control. We consider a PD controller and a model based controller. Although the system considered is from the simplest possible robot, it is still very difficult to do an analytical study. The corresponding dynamical system is of dimension $\mathbf{R}^{4}$ and is non-autonomous. Only a numerical study appears to be feasible.

The organization of the paper is as follows: we first develop the dynamical system to be studied and list a few analytical results, then describe the numerical study done, then follow up with a discussion of the results and finally present our conclusion

\section{Dynamic Equations of a 2R Robot}

The dynamics of a $2 R$, rigid, planar manipulator can be modeled as in[2] :

$$
\mathbf{M}(\theta) \ddot{\theta}+\mathbf{C}(\theta, \dot{\theta})=\tau
$$

where, $\theta(t)$ is the $2 \times 1$ vector of joint angles, $\mathrm{M}(\theta)$ is the mass matrix, $\mathbf{C}(\theta, \dot{\theta})$ is the $2 \times 1$ vector of Coriolis and centrifugal torques, and $\tau$ is the vector of joint torques.

Equation (1) can be represented in state space form as

$$
\begin{aligned}
\dot{x}_{1}= & x_{2} \\
\dot{x}_{2}= & \left(1 / P_{3}\left(x_{3}\right)\right)\left\{K _ { 3 } ( x _ { 3 } ) \left(K_{2}\left(x_{3}\right) x_{2}^{2}+\right.\right. \\
& \left.\left.N_{2}\left(2 x_{2} x_{4}+x_{4}^{2}\right)\right)+N_{2} \tau_{1}-K_{2}\left(x_{3}\right) \tau_{2}\right\} \\
\dot{x}_{3}= & x_{4} \\
\dot{x}_{4}= & \left(1 / P_{3}\left(x_{3}\right)\right)\left\{-K_{3}\left(x_{3}\right)\left(K_{1}\left(x_{3}\right) x_{2}^{2}\right.\right. \\
& \left.+K_{2}\left(x_{3}\right)\left(2 x_{2} x_{4}+x_{4}^{2}\right)\right)-K_{2}\left(x_{3}\right) \tau_{1} \\
& \left.+K_{1}\left(x_{3}\right) \tau_{2}\right\}
\end{aligned}
$$

where, the state variables are the joint variables $\theta_{1}, \theta_{2}$ and their derivatives, and

$$
\begin{aligned}
P_{3}\left(x_{3}\right)= & \operatorname{det}\left[\mathbf{M}\left(x_{3}\right)\right] \\
K_{1}\left(x_{3}\right)= & m_{1} r_{1}^{2}+I_{1}+I_{2}+m_{2} r_{2}^{2}+m_{2} l_{1}^{2} \\
& +2 m_{2} l_{1} r_{2} \cos \left(x_{3}\right) \\
K_{2}\left(x_{3}\right)= & m_{2} r_{2}^{2}+I_{2}+m_{2} l_{1} r_{2} \cos \left(x_{3}\right) \\
K_{3}\left(x_{3}\right)= & m_{2} l_{1} r_{2} \sin \left(x_{3}\right) \\
N_{2}= & I_{2}+m_{2} r_{2}^{2}
\end{aligned}
$$

In the above equations, $m_{i}, l_{i}, I_{i}$ and $r_{i}$ are the mass, length, inertia and location of the center of gravity of link $i$ respectively. Figure 1 shows a sketch of the $2 \mathrm{R}$ robot under consideration.

\section{Feedback Control of a 2R Robot}

We consider the following two well known control laws[2], namely, (i) Proportional Derivative (PD) Control and (ii) Model Based Control.

For the PD controller, the torque at the joint $i$ is calculated as

$$
\tau_{i}=\ddot{\theta}_{d_{i}}+K_{v_{i}}\left(\dot{\theta}_{d_{i}}(t)-\dot{\theta}_{i}(t)\right)+K_{p_{i}}\left(\theta_{d_{i}}(t)-\theta_{i}(t)\right)
$$

where, $\theta_{d_{i}}(t)$ is the desired periodic trajectory to be tracked in joint space, $K_{p_{i}}$ and $K_{v_{i}}$ are the positive proportional and derivative gains.

It can be shown that, in the absence of gravity, the PD control law achieves asymptotic tracking of the desired joint positions $[10,11]$. Our aim in this paper is to study the global behavior of the $2 \mathrm{R}$ robot when the controller gains, namely $\mathbf{K}_{p}$ and $\mathbf{K}_{v}$, are varied.

The desired repetitive trajectories, in the joint space, are described by

$$
\begin{aligned}
& \theta_{d 1}=A_{1} \sin (\omega t) \\
& \theta_{d 2}=A_{2} \sin (\omega t)
\end{aligned}
$$

Substitution of equations (4) and (5) into equation (2) results in a system of four, first order, ordinary differential equations which are coupled, nonlinear and non-autonomous.

For the model based control, we follow Craig[2] and calculate the joint torques as

$$
\tau=\widehat{\mathbf{M}}(\theta) \tau_{p}+\widehat{\mathbf{C}}(\theta, \dot{\theta})
$$

where, $\tau_{p}=\ddot{\theta}_{d}+\mathbf{K}_{v}\left(\dot{\theta}_{d}-\dot{\theta}\right)+\mathbf{K}_{p}\left(\theta_{d}-\theta\right), \widehat{\mathbf{M}}(\theta)$ is the estimated mass matrix, $\widehat{\mathbf{C}}(\theta, \dot{\theta})$ is the estimated Coriolis and centrifugal torques vector, and $\mathbf{K}_{p}, \mathbf{K}_{v}$ are $2 \times 2$ constant, diagonal gain matrices. The estimates, $\widehat{\mathbf{M}}(\theta)$ and $\widehat{\mathbf{C}}(\theta, \dot{\theta})$, are computed by perturbing the robot parameters as follows:

$$
\begin{aligned}
\widehat{m}_{i} & =(1+\epsilon) m_{i} \\
\widehat{r}_{i} & =(1+\epsilon) r_{i} \\
\widehat{I}_{i} & =(1+\epsilon) I_{i} \\
\widehat{l}_{i} & =l_{i}
\end{aligned}
$$

where $\epsilon>0$ implies an overestimated model and $-1<\epsilon<0$ implies an underestimated model. For $\epsilon=0.0$, the closed-loop error equation become linear second order ordinary differential equations, and they can never exhibit chaos.

Craig[12] has given a robustness conjecture for the model based, computed torque, scheme which states that if $\widehat{\mathbf{M}}(\theta)>0$ and symmetric and $\mathbf{K}_{v}>\alpha * \mathbf{I}_{n}$, then the system is $L_{\infty}$ stable. As far as we are aware there exists no proof of this conjecture in literature. 
In this paper, we examine the the global behavior relative to the control parameters of the $2 \mathrm{R}$ robot with model based control scheme. The desired trajectories are again given by equation (5).

Substitution of equations (5) and (6) in equations (2) results in a system of four first order ordinary differential equations which are coupled, nonlinear and non-autonomous.

The two dynamical systems, obtained for the PD and the model based controller, have trigonometric nonlinearities and are dissipative. The amount of dissipation is determined by the velocity gain $\mathbf{K}_{v}$. The $\mathbf{R}^{4}$ state space ( $\mathbf{R}^{5}$ in the case of the corresponding autonomous system) makes it very difficult for analytical work. One can show that the only fixed point of this set of differential equations is $(0,0,0,0)$ and it is difficult to make any more general statements.

The above systems are much more complicated compared to the systems studied in chaos literature which are usually of dimensions less than four, and usually with nonlinearities which facilitate some analytical study. One way to study these systems is by a digital computer. In the next section, we present the details of the numerical study done for the above systems.

\section{Numerical Study}

To perform a numerical study on the two nonlinear, non-autonomous, ordinary differential equations, representing feedback control of a planar $2 \mathrm{R}$ robot, we have chosen the Denavit-Hartenberg and inertial parameters of the first two links of the CMU DD Arm II[13]. Figure 1 shows a sketch of the 2R robot with all its parameters.

As mentioned before, we are interested in global behavior when the controller gains are varied. In general there would be 4 controller gains $-K_{p}$ and $K_{v}$ for each of the two joints. To make the search space smaller we have assumed that the gains are same for both joints.

The state equations were integrated numerically by a variable step, variable order, predictor corrector Adams algorithm[14]. In order to ensure that the numerical plots were not an artifact of the numerical integration scheme, the results were verified with Runge-Kutta 5-6[15] integration routine. The results were also checked for relative and absolute error tolerances of $10^{-6}$ and $10^{-9}$.

To study the global behavior of the feedback controlled $2 R$ robot under periodic desired trajectories, we first ignore the transients and then look at the following:

a) Phase plots - phase plots are plots of joint velocity versus joint positions. The actual flow is in $\mathbf{R}^{4}$, but we plot projections in $\mathbb{R}^{2}$. These plots quickly give an idea if the orbits are periodic or not. b) Poincaré section - The integration results are sampled at the forcing frequency(in our case 2.0 $\mathrm{rad} / \mathrm{s}$ ). The Poincaré Map is 4 dimensional, but we show $\mathbf{R}^{2}$ projections. It is difficult to obtain or visualize the fractal structure of the map from the projections.

(c) The largest Lyapunov exponent was calculated using the algorithm given by Wolf et al.[16]. The Lyapunov exponent is a measure of the sensitivity of the system to changes in initial conditions. Conceptually, one can imagine a small sphere of initial conditions in phase space and look at its deformation into an ellipsoid under the dynamics of the system. If $d$ is the maximum length of the ellipsoid and $d_{0}$ the initial size of the initial condition sphere, the Lyapunov exponent, $\lambda$, is given by the equation

$$
d(t)=d_{0} e^{\lambda\left(t-t_{0}\right)}
$$

The average of $\lambda$ over the phase space can be represented as

$$
\lambda=\lim _{N \rightarrow \infty} \frac{1}{N} \sum_{i=1}^{N} \frac{1}{t_{i}-t_{0 i}} \log \left(d_{i} / d_{0 i}\right)
$$

An N-dimensional system will have N Lyapunov exponents. A positive exponent implies chaotic dynamics.

A search was done by varying the gains $K_{p}$ and $K_{v}$ and calculating the largest Lyapunov exponent. In case of model based control, the measure of mismatch, $\epsilon$, was also varied.

(d) Bifurcation diagrams - the phenomena of sudden change in the motion as a parameter is varied is called a bifurcation. A bifurcation diagram is a technique for examining the prechaotic (route to chaos) or post chaotic changes in a dynamical system under parameter variations. Bifurcation diagrams were computed using the brute force algorithm as described in [17].

\section{$5 \quad$ Results and Discussion}

A numerical study of the feedback control equations for the two control algorithms was done taking the desired repetitive trajectory to have $A_{1}=\pi / 2$ $\mathrm{rad}, A_{2}=\pi / 4 \mathrm{rad}$, and $\omega=2.0 \mathrm{rad} / \mathrm{s}$. The simulations were performed with different initial conditions and the same qualitative behavior was observed after neglecting the initial transients. Figure 2 shows the phase plots for non-chaotic and chaotic parameters. It can be observed that in the non-chaotic case the trajectory settles to a limit cycle whereas in the chaotic case the trajectory moves about in a bounded region in the phase space known as the attractor. It 
is to be noted that what we see in the phase plots is only a projection of the actual flow which is in $\mathbf{R}^{4}$.

Figure 3 shows Poincaré section for chaotic parameters of figure 2. The fractal nature is visible even though it is only a projection. Figures 4 and 5 show plots of the parameters for which the system was found to be chaotic. The values of $K_{p}$ and $K_{v}$ giving rise to chaos are marked by the symbol *. A plot of the Lyapunov exponent for a particular set of chaotic parameters is also shown. These plots were obtained by a numerical search in the $\left(K_{p}, K_{v}\right)$ space with $K_{p}$ and $K_{v}$ varied in steps of 1.0 . It can be seen that chaos occurs only for small gains and, in particular, for highly underdamped systems with small values of $K_{v}$. More detailed regions of the chaotic parameter space can be obtained by taking smaller steps in the scan.

In the case of model based control $\epsilon$ was varied in steps of 0.05 , and $K_{p}, K_{v}$ by 1.0 . It was found that chaotic behavior was seen only when the mismatch parameter, $\epsilon$, was large(more than 0.6) and chaos was more easily seen for underestimations. For overestimations, chaotic behavior was observed only for very small values of $K_{p}$ and $K_{v}$. This can be explained by realising that the 'effective' closed-loop gains are given by $\mathbf{M}^{-1} \widehat{\mathbf{M}} \mathbf{K}_{p}$ and $\mathbf{M}^{-1} \widehat{\mathbf{M}} \mathbf{K}_{v}$. When $\epsilon>0$, the 'effective' gains become large and when $\epsilon<0$ 'effective' gains become small.

Figure 6 shows bifurcation diagrams of state variables $x_{1}$ and $x_{3}$ for two sets of parameters. A bifurcation from period one to period two can be clearly seen. Again, it must be noted that the figures are a projection of the trajectory bifurcating in $\mathbf{R}^{4}$.

\section{Conclusions}

In this paper, we have demonstrated that the non-linear, ordinary differential equations describing the motion of a feedback controlled, rigid, planar, $2 \mathrm{R}$ robot undergoing repetitive motions can exhibit chaotic motions. Unlike most chaotic systems with non-linear springs, the non-linearity in this paper are trigonometric in nature arising from the fact that the inertia matrix of a $2 \mathrm{R}$ robot is dependent on its configuration. Unlike most systems studied by chaos researchers, the state equations for feedback controlled robot are 4 dimensional and non-autonomous and hence only a numerical study is feasible. From the numerical study presented in this paper we present the following major conclusions:

1) Chaotic motions can be seen both for a simple PD controller and for a model based controller with mismatch in model parameters. The existence of chaotic motions were verified by careful numerical simulations and by use of the largest Lyapunov exponent.
2) Chaotic motions occur for small values of the derivative gains and for large mismatch between the dynamic model and the actual robot parameters. Chaotic motions are only seen if the system is grossly underdamped. The chaotic motions are seen more easily for underestimated models. For overestimated models, chaotic motions are seen for extremely low values of controller gains. This can be explained by the observation that the effective closed loop gains for an overestimated model is larger than the proportional and derivative gains.

3) The route to chaos appears to be through period doubling. However this requires further study.

Although the range of controller gains, in particular the derivative gains, is far removed from critically damped(or overdamped) regime in any actual robot, this study, apart from being of mathematical interest, can give lower bounds on controller gains. The study can also help in obtaining conditions for better trajectory tracking in feedback controlled robots.

\section{References}

[1] Moon F. C. 1987. Chaotic Vibrations, John Wiley and Sons.

[2] Craig J. J. 1989. Introduction to Robotics, Mechanics and Control, Addison-Wesley.

[3] Guckenheimer J., Holmes P., 1983. Nonlinear Oscillations, Dynamical Systems, and Bifurcations of Vector Fields, Springer-Verlag.

[4] Streit D. A., Krousgrill C. M., Bajaj A. K., September 1989. Nonlinear Response of Flexible Robotic Manipulators Performing Repetitive Tasks, Transactions of the ASME, Vol. 111, pp. $470-480$.

[5] Bühler M., Koditschek D. E., 1990. From Stable to Chaotic Juggling : Theory, Simulation, and Experiments, Proceedings of IEEE International Conference on Robotics and Automation, Cincinatti, USA, pp. 1976-1981.

[6] Vakakis A. F., Burdick J. W., 1990. Chaotic Motions in the Dynamics of a Hopping Robot, Proceedings of IEEE International Conference on Robotics and Automation, Cincinatti, USA, pp. 1464-1469.

[7] M'Closkey R. T., Burdick J. W., 1993. Periodic Motions of a Hopping Robot with Vertical and Forward Motion, International Journal of Robotics Research, Vol. 12(3), pp.197-218 
[8] Mahout V., Lopez P., Carcassés J. P., Mira C. 1992. Complex Responses(Chaotic) of a TwoRevolute Joints Robot for Periodical Torque Inputs, IFToMM-jc International Symposium on Theory of Machines and Mechanisms, Nagoya, JAPAN

[9] Mahout V., Lopez P., Carcassés J. P., Mira C. 1992. Complex Behaviours of a Two-Revolute Joints Robot: harmonic, subharmonic, higher harmonic, fractional harmonic, chaotic responses. IFToMM-jc International Symposium on Theory of Machines and Mechanisms, Nagoya, JAPAN

[10] Asada H., Slotline J. J. E., 1986. Robot Analysis and Control, John Wiley and Sons.

[11] Spong M. W., Vidyasagar M., 1989. Robot Dynamics and Control, John Wiley and Sons.

[12] Craig J. J.,1988. Adaptive Control of Mechanical Manipulators, Addison-Wesley.

[13] Khosla P. K., 1986. Real-Time Control and Identification of Direct-Drive Manipulators, $\mathrm{Ph}$. D. Thesis, Dept. of Electrical and Computer Engg., Carnegie Mellon University, USA.

[14] Gordon M. K., Shampine L. F. 1975. Computer Solutions of Ordinary Differential Equations: The Initial Value Problem, W. H. Freeman $\&$ Co.

[15] User's Manual IMSL Math/Library, 1989.

[16] Wolf A., Swift J. B. Swinney H. A., Vastano J. A.,1985. Determining Lyapunov Exponents from a Time Series, Physica 16D, 16D:285-317.

[17] Parker T. S., Chua L. O., 1989. Practical Numerical Algorithms for Chaotic Systems, Springer Verlag.

Physical parameters of the 2R robot

\begin{tabular}{|c|c|c|c|c|}
\hline Link & $\begin{array}{c}\text { Length } \\
(\mathrm{m})\end{array}$ & $\begin{array}{c}\text { Mass } \\
(\mathrm{kg})\end{array}$ & $\begin{array}{c}\text { C.G. } \\
(\mathrm{m})\end{array}$ & $\begin{array}{c}\text { Inertia } \\
\left(\mathrm{kgm}^{2}\right)\end{array}$ \\
\hline 1 & 0.5 & 20.15 & 0.18 & 6.3 \\
\hline 2 & 0.4 & 8.25 & 0.26 & 1.64 \\
\hline
\end{tabular}

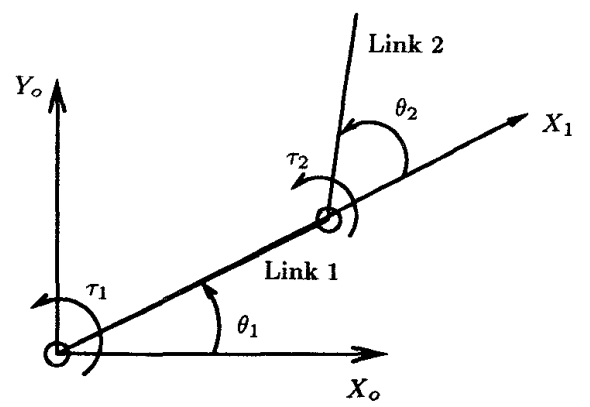

Figure 1: A schematic of a 2R planar rigid robot.
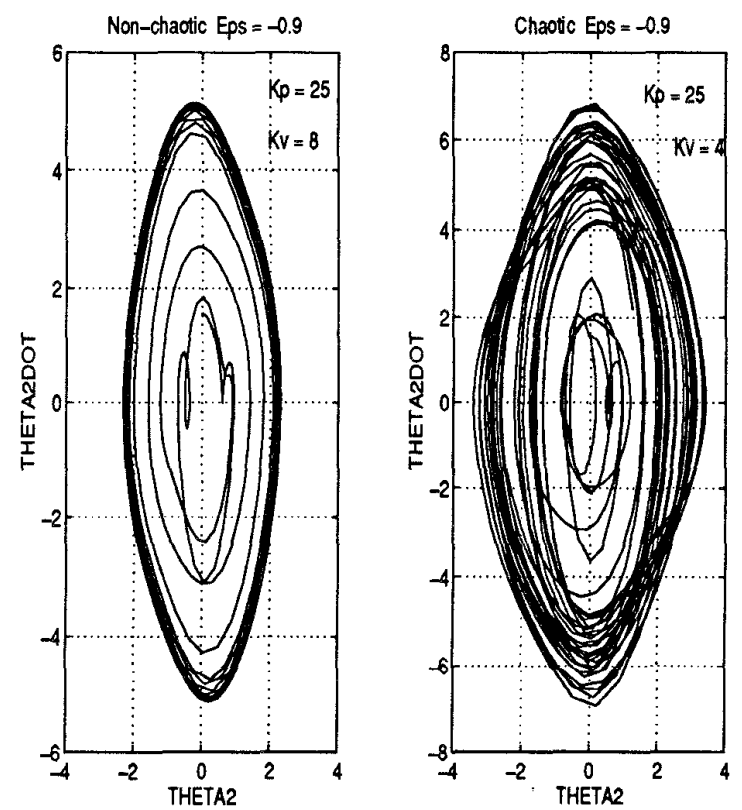

Figure 2: Non-chaotic and chaotic phase plots. 

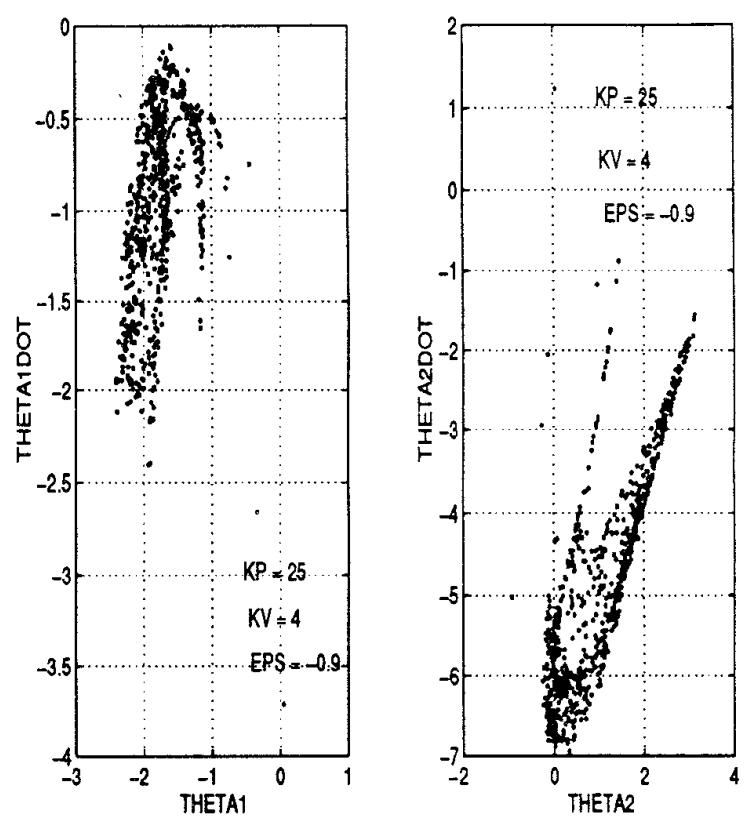

Figure 3: Poincaré maps.
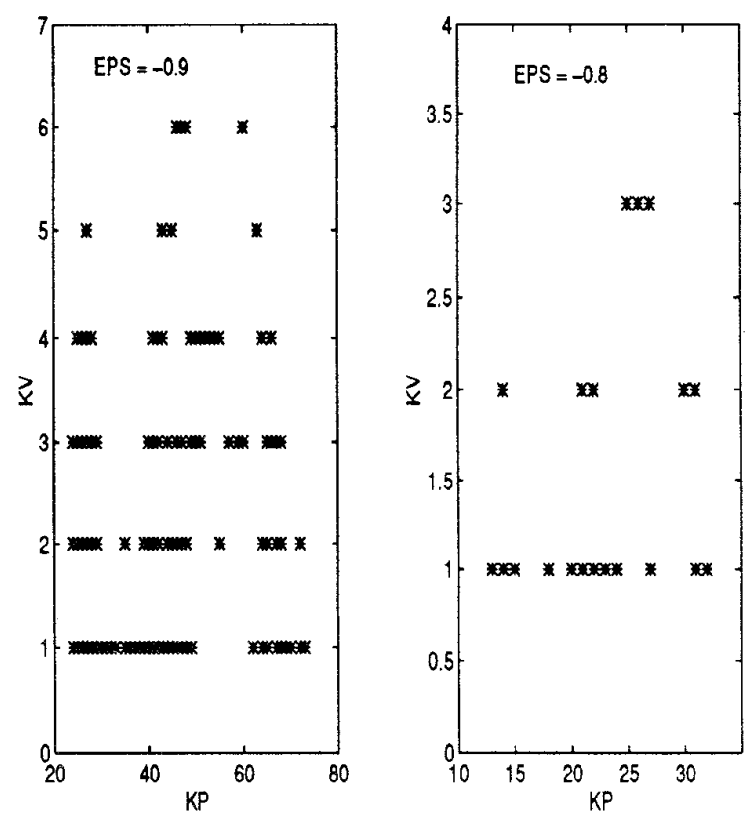

Figure 4: Regions of chaos for the model-based controller.
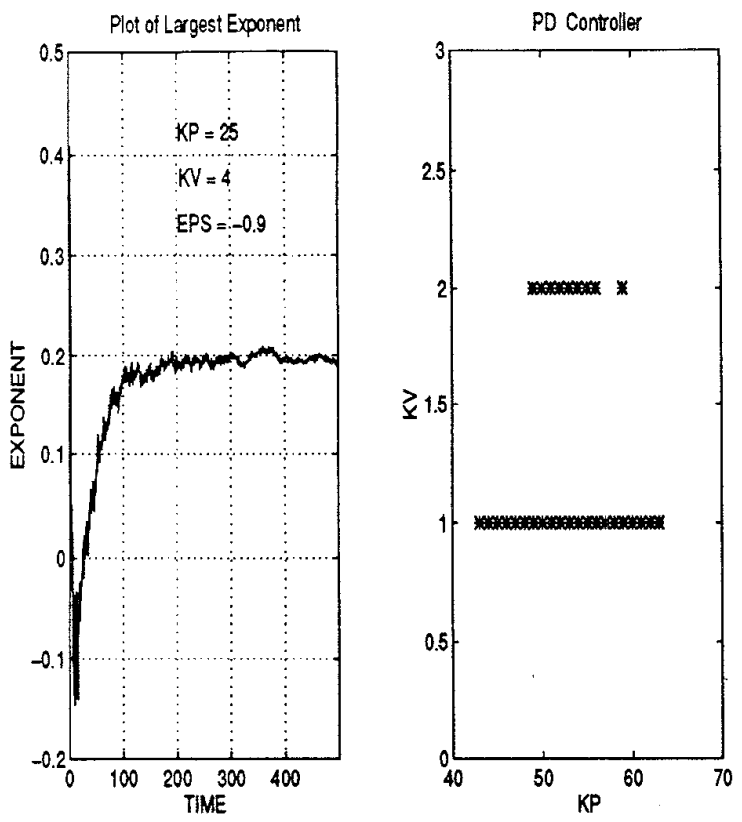

Figure 5: Largest Lyapunov exponent for the modelbased controller, and regions of chaos for the PD controller.
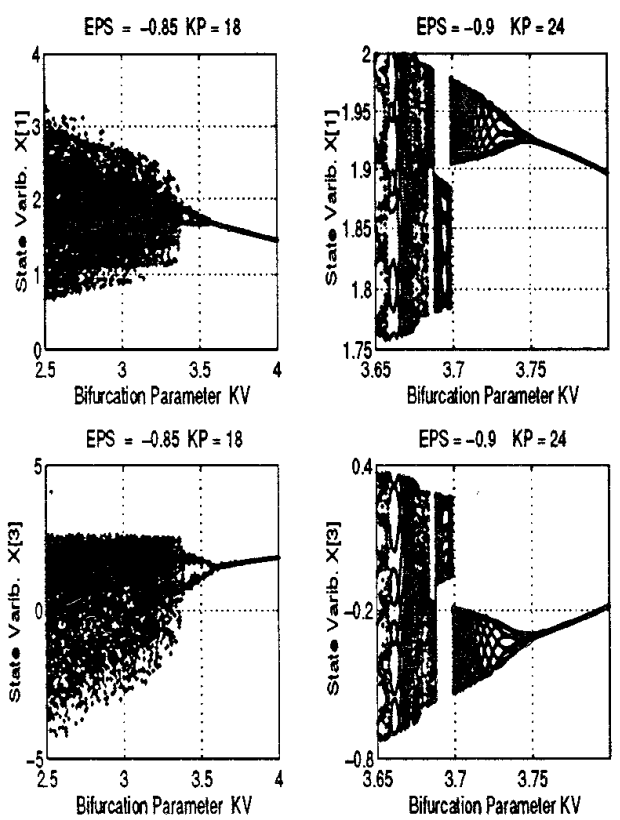

Figure 6: Bifurcation diagrams for the model-based controller. 